\title{
Sub-wavelength detection with waveguides in optical recording
}

\author{
S. Musa ${ }^{a *}$ S.F. Pereira ${ }^{a}$, and J.J.M. Braat ${ }^{\mathrm{a}}$ \\ F. Fransoo ${ }^{\mathrm{b}}$, D. Van Thourhout ${ }^{\mathrm{b}}$, and R. Baets ${ }^{\mathrm{b}}$

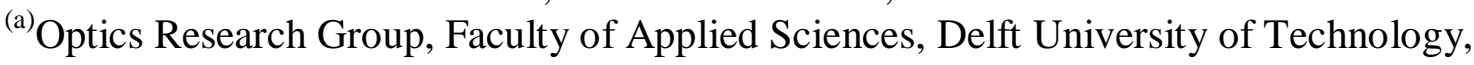 \\ Lorentzweg1, 2628 CJ Delft, The Netherlands \\ ${ }^{(b)}$ Ghent University-IMEC, Department of Information Technology (INTEC) \\ Sint-Pietersnieuwstraat 41, 9000 Gent, Belgium
}

\begin{abstract}
We present a study on the use of optical waveguides in readout units for optical recording. Optical waveguides can be used to increase the storage capacity of optical recording, which is limited by diffraction. Optical waveguides are attractive for application in readout units of optical recording systems because it is possible to make compact configurations where the amplitude and the phase of an optical signal can be detected. We propose a couple of different detection schemes for reading out data recorded in an optical disc using waveguides. The potential of these detection methods has been investigated using numerical calculations, which are presented and discussed.
\end{abstract}

Keywords: Optical recording, Sub-wavelength imaging, Optical waveguides

\section{INTRODUCTION}

The evolution of optical data recording from the CD-ROM to Blu-Ray system is characterized by a continuous quest for higher recording density, while the attractive properties of an optical system such as robustness, cost effectiveness and exchangeability are maintained. Increasing the storage density beyond the recent developed Blu-Ray system is hampered by technical difficulties, which include laser wavelength in the ultraviolet region and extremely high numerical apertures. In order to achieve higher recording density, there is a need for alternative detection methods that can circumvent these challenges and retain the attractive properties of the developed optical system. Techniques such as "near field" and "solid immersion lens" readout have shown the prospect of increased storage density, however, they are not exchangeable [1].

In this work, we propose a new approach to detect information from an optical disc, which involves the use of optical waveguides to illuminate and read the optical disc [2,3]. This method is expected to give very high resolution as optical waveguides are sensitive to both the phase and the amplitude of the incoming signal. Moreover, by using integrated optics, a very compact and robust read-out system could be realized. In the following, the basic principle of using waveguides as sub-wavelength probes will be explained. After that we will outline different detection methods. Simulation results will be presented and discussed.

\section{DETECTION WITH WAVEGUIDES}

It is well known from the theoretical point of view that an increase in resolution in a noiseless system can be achieved by, for example, reconstruction of the object field [4]. This is based on the detection of the complete field (including the phase and the amplitude) originating from an illuminated restricted object area. However, experimentally, this unlimited resolution is very difficult to be reached (if not impossible), since the reconstruction of an arbitrary object can only be achieved if perfect knowledge of the field is available. In particular, noise hampers the possibility of determining the field in a unique way. Investigations examining the use of non-classical light in order to obtain a gain in the resolution by "eliminating" the classical noise are being considered, but though very interesting, they involve difficult experimental achievements and convincing demonstrations are still far from being realized [5].

If one forgets about reconstructing an arbitrary object, the reconstruction becomes feasible if some a-priori knowledge of the object being investigated is known. The use of a-priori information combined with a new phase and amplitude

\footnotetext{
*e.mail address: s.musa@tnw.tudelft.nl
} 
detection form the basis of the problem that we are investigating. In our case, the object is an isolated bit pattern composed of $\mathrm{n}$ pits and the goal is to uniquely characterize all $2^{\mathrm{n}}$ possible bit patterns by carefully measuring the field reflected from a given bit pattern. This detection must be capable of reasonably isolating the bit pattern from the neighbouring area and should be sensitive to the phase and the amplitude of the field. Optical waveguides are an interesting option in this case, since they have a well-defined numerical aperture and it is possible to build a compact and robust head where both the amplitude and the phase can be detected as will become clear later.

The complex amplitude of an injected field in a waveguide is proportional to the normalized value of the overlap integral of the field and the particular considered mode of the guide:

$$
a_{m}=\frac{\int \psi(x, y) \varphi_{m}^{*}(x, y) d x d y}{\sqrt{\int|\psi(x, y)|^{2} d x}}=A_{m} \exp \left(j \alpha_{m}\right),
$$

where $\psi(x, y)$ is the field originating from an illuminated bit pattern at the interface of the waveguide, $\varphi_{m}(x, y)$ is a modal field of a waveguide, $A_{m}$ is the amplitude and $\alpha_{m}$ is a phase factor. The above expression shows that the modal fields carry information about both the amplitude and the phase of the field picked up by the waveguide. Given a-priori knowledge of the field, by simultaneous measurements of the amplitudes and the phases of modes propagating in an array of waveguides or in a multimode waveguide intercepting the reflected field, the profile of this field can be accurately synthesised. Apart from detecting the complete field, gain in the resolution can be achieved with waveguides by detecting either the amplitude or the phase. When only the amplitude is used, the reading out of data can be done in two different ways. In one method the waveguide can be scanned over continuos tracks of bit-patterns while the intensities of the light coupled to the different modes of the waveguide will be monitored to extract information about the data on the disc. In the second method the waveguide is used to illuminate and detect an isolated bit-pattern. The intensities coupled to each mode of the waveguide combined with an a-priori knowledge of the bit-pattern are used to identify the bit-pattern. Both methods show interesting results as will be discussed later in more details.

\section{AMPLITUDE DETECTION}

\subsection{Continuous scanning of the amplitude}

In 0 two different configurations of the proposed waveguide-based amplitude detection systems are given. Note that the data is represented as a continuous track of bit patterns over which the waveguide can be scanned. In the configuration depicted in Fig. 1a the waveguide is placed further away (typically hundreds of wavelengths) from the disc and light is focused on the disc by an optical lens. We will refer to this configuration as far-field detection system. In the configuration shown in Fig. 1b, the waveguide is placed close to the disc ( 0.25 to 1 wavelength) and we will refer to it as intermediate- or near-field detection system.

\section{Far-field detection system}
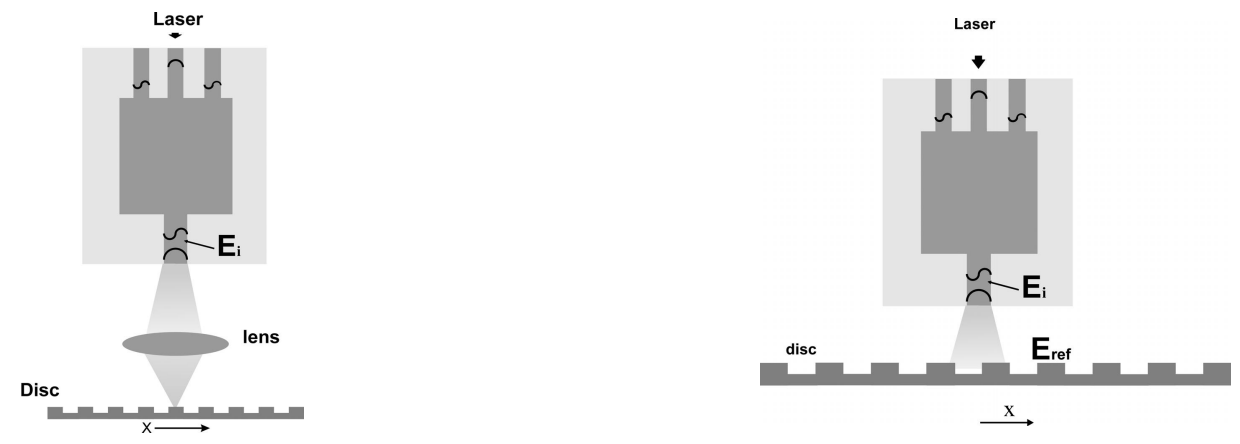

Fi.g. 1 Basic layout of waveguide read-out unit. (a) far-field and (b) intermediate-field configurations

In this system light from one mode or a linear combination of waveguide modes is focused by an optical lens on the disc. The spot is scanned through a track of bumps or holes representing the information printed on the disc (see Fig. 1a). The 
modulated reflected light couples into the different modes of the waveguide, and the intensity of the light coupled to each mode can be measured by separating the modes using an integrated optical mode splitter. In this way one obtains time-dependent signals which are related to the scanned bit pattern; in practice we detect the $0^{\text {th }}$ and the $1^{\text {st }}$ order modes and two distinct signals become available. The far-field configuration is similar to the present CD system and the waveguide acts as dual mode detector.

\section{Intermediate-field detection system}

ob shows a basic layout of an intermediate- or near-field optical readout head. In this system the waveguide is placed close to the disc so that no focusing lens is used. The gap distance between waveguide and disc is in the order of the wavelength. Light reflected from the bit pattern couples back to the different modes of the waveguide. The processing of the detected signal is done by parameter extraction of bits on track as in the far-field case.

\section{Parameter extraction of bits on a track}

\section{Distorted signal at detectors $2 \times 2^{\mathrm{N}}$ calculated responses}

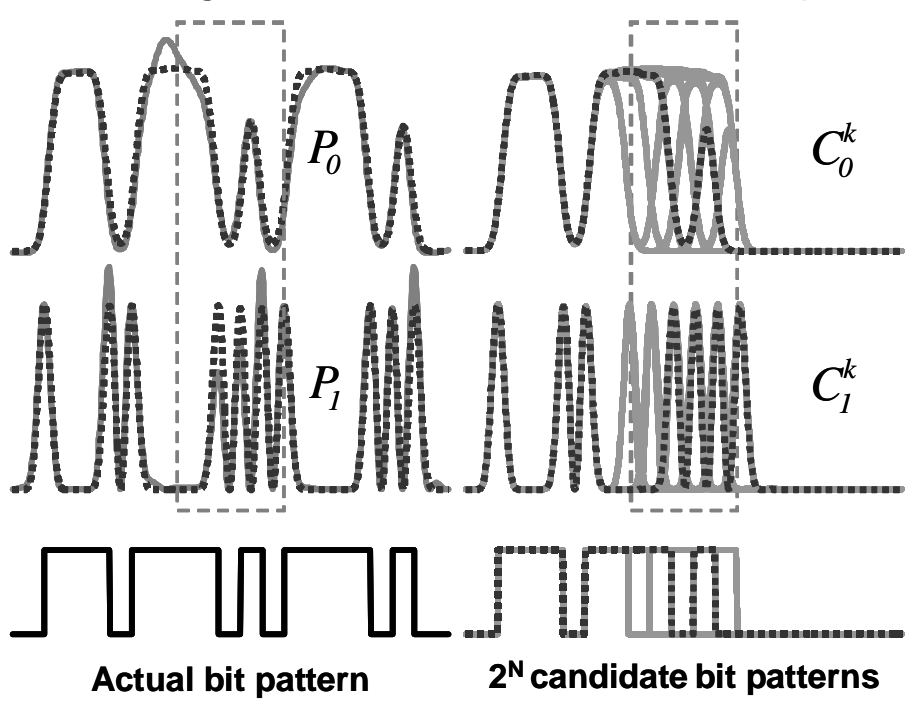

Fig. 2 On the left the original bit pattern and the detected signals are shown. On the right the candidate bit patterns and the calculated responses are shown. $\mathrm{C}_{0}{ }^{\mathrm{k}}$ and $\mathrm{C}_{0}{ }^{\mathrm{k}}$ are the calculated powers which coupled from the $\mathrm{k}^{\text {th }}$ bit pattern to the $0^{\text {th }}$ and $1^{\text {st }}$ order modes, respectively.

By scanning the disc, the $0^{\text {th }}$ and the $1^{\text {st }}$ order modes pick up different signals from the bit patterns. Fig. 2 shows these detected signals, distorted by noise and crosstalk. If one chooses a few bits as parameters, one can calculate the responses for a few candidate bit patterns. The detected signals can be compared with theoretically calculated signals and by parameter extraction the original bit patterns can be recovered. The novelty within this approach is that the first order and zeroth order signals are detected simultaneously and information from both signals can be used to find the correct bit pattern.

\section{Point response and frequency response}

In order to analyse the limitations of this detection system and compare its performance with that of existing systems one can use point and frequency response functions. We limit the analysis to the 1-D case. The waveguide scanning system is a coherent detection system. This means that the complex excitation coefficients of the zeroth- and the first-order modes, $a_{0}$ and $a_{l}$, are functions of the complex reflection function of the disc, $r(x)$, and the scanning position $x_{s}$. The power detected at the detector is proportional to the squared amplitude of this mode excitation.

$$
a_{m}\left(x_{s}\right)=\int_{-\infty}^{\infty} r\left(x-x_{s}, y\right) p s f_{m}(x) d x \text { and } P_{m}\left(x_{s}\right)=\left|a_{m}\left(x_{s}\right)\right|^{2}
$$


with $\operatorname{psf}_{m}(x)$ is called the point response. This function should not be confused with a similar, but not equal, point response function defined in incoherent optical systems. The coherent frequency response, $\operatorname{MTF}_{m}\left(v_{x}\right)$, is defined as the Fourier transform of the point response $p s f_{m}(x)$.

$$
\operatorname{MTF}_{\mathrm{m}}\left(v_{x}\right)=\int_{-\infty}^{\infty} p s f_{m}(x) \exp \left(2 \pi j x v_{x}\right) d x
$$

These frequency responses for the zeroth- and for the first-order modes are shown in Fig. 3. The frequency response gives a clear idea of which spatial frequencies on the disc are detected and which are not. As one sees, the diffraction barrier of $2 N A / \lambda$ is not broken, unless the gap between waveguide and disc in the intermediate field is smaller than half wavelength. The frequency response of the zeroth order mode in the far field system is almost identical to that of a confocal microscope. The frequency response of the first order mode shows a drop at the lower spatial frequencies, which means that this mode has a relative higher sensitivity at the higher spatial frequencies as compared to the zerothorder mode. The information acquired from the first-order mode, comparable to the well-known Push-Pull signal, can be used, for example, to reduce bit error rate at high frequencies due to the noise [6].
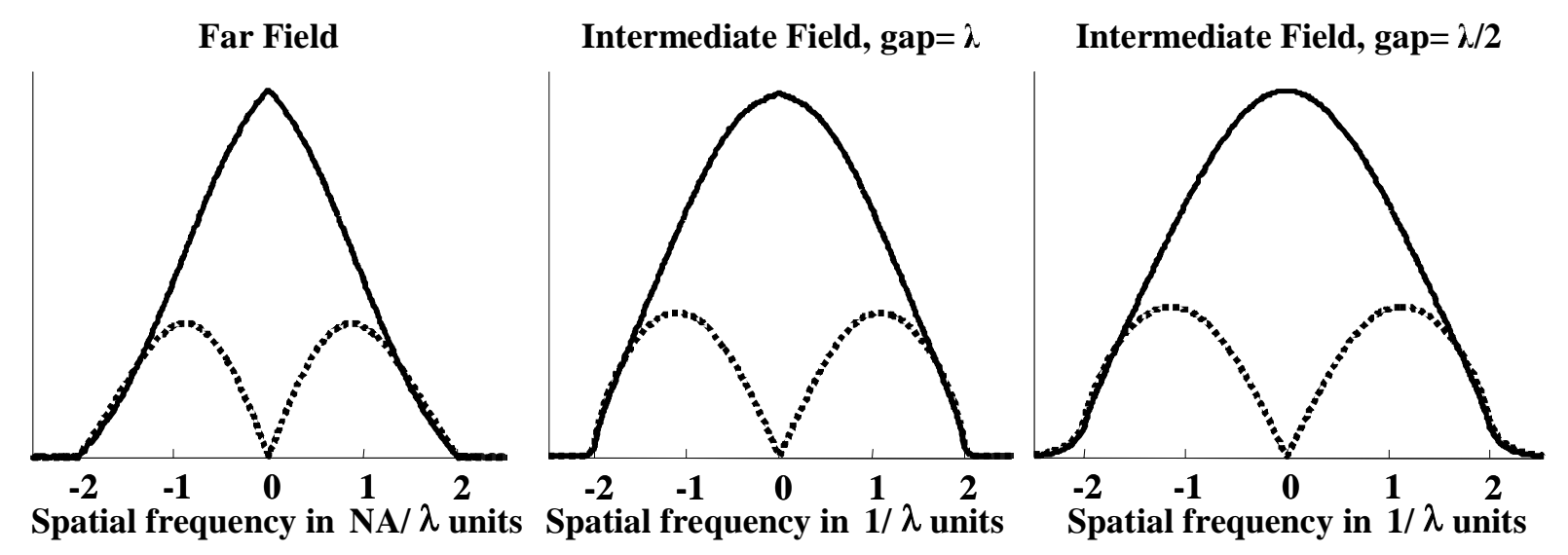

Fig. 3 Frequency responses for the zeroth- and the first-order modes, shown in solid and dashed lines, respectively.

\subsection{Discrete detection of the amplitude}

In order to utilise amplitude information, combined with a-priori knowledge of the bit pattern, to recover bit patterns with features smaller than $\lambda / 4 N A$ (the halve of the period of the minimal period according to the diffraction limit), we have used discrete detection of isolated bit patterns. Fig. 4 shows a basic layout of an optical read-out head used for discrete detection of the amplitude. This system is similar to the intermediate- or near-field detection system depicted in Fig. 1b, with the exception that the data on the disc is not registered in continuous tracks but in isolated groups of bit patterns as illustrated in Fig. 4. This specific way in which the data is recorded on the disc requires different methods for the illumination of the bit-pattern and the processing of the detected signals. The laser light is coupled to the waveguide, which subsequently illuminates an isolated binary bit pattern on the optical disc. The bit pattern consists of $n$ pits with inter-pit distances smaller than the diffraction limit (half a wavelength). Light reflected from the bit pattern couples back to the different modes of the waveguide; these modes are split inside the mode splitter and are measured accordingly. Here for simplification, we consider only the $0^{\text {th }}$ and $1^{\text {st }}$ order modes (also called TE0 and TE1 modes). 


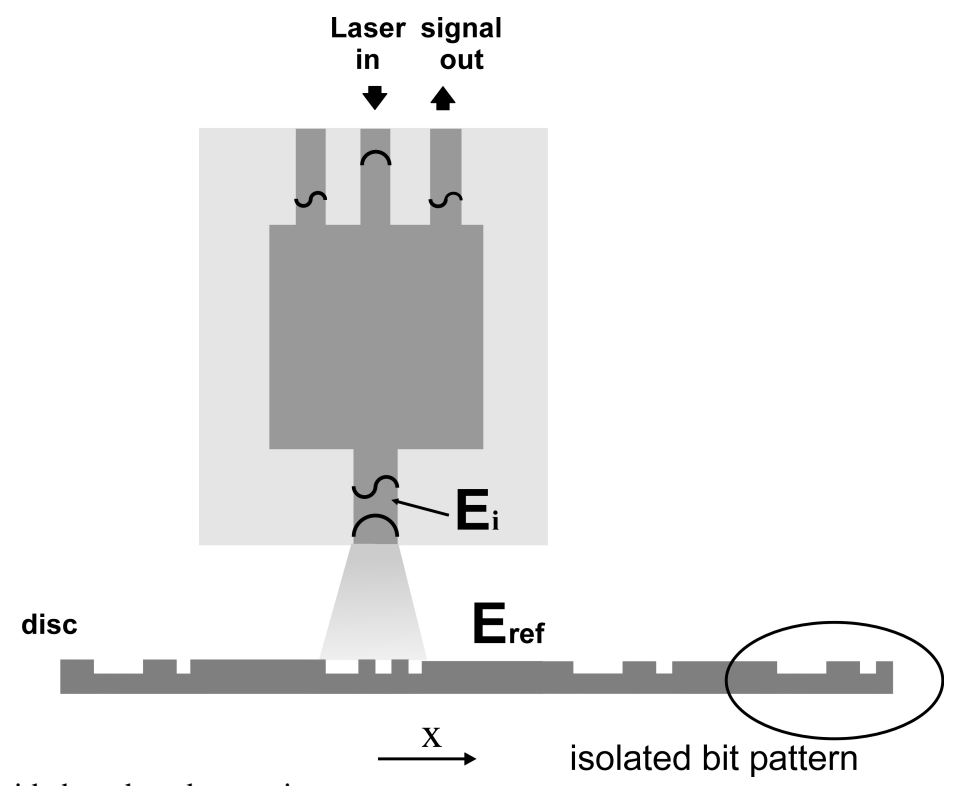

Fig. 4 Basic layout of waveguide based read-out unit.

If the waveguide is placed at a distance of the order of 0.5 to 1 wavelength with respect to the disc, there is a considerable level of discrimination in the wavefront, which is reflected from different bit patterns. Since the waveguide is sensitive to both the amplitude and phase of the incoming light, the intensity coupled to each mode will be different for each bit pattern. The intensity of the light coupled to the individual modes can be individually measured. As the waveguide is scanned along a track of isolated bit pattens one or more measurements can be taken per bit pattern. Discrete measurements per bit pattern can be done, for example, by modulating the laser used for the illumination. In each measurement, a set of the intensities measured for the different waveguide modes can be used to identify the bit pattern.

We have calculated the intensity coupled into the two modes, TE0 and TE1, of a bimodal waveguide from a set of illuminated asymmetric bit patterns. Each bit pattern consists of 5 pits where inter-pit distance of the first three pits on the left side is $0.2 \lambda$ and of the pits on right side is $0.15 \lambda$. In Fig. 5, the power coupled to the TE0 mode is plotted against the power coupled to the TE1 mode, where each point corresponds to one of the $2^{5}$ possible bit patterns. This plot shows that even though the pits are smaller than $0.5 \lambda$, the combination of the $0^{\text {th }}$ and $1^{\text {st }}$ order modes makes possible to distinguish all bit patterns. The limitation of the highest obtainable resolution will depend on the level of the system noise, which is not yet considered in this calculation.

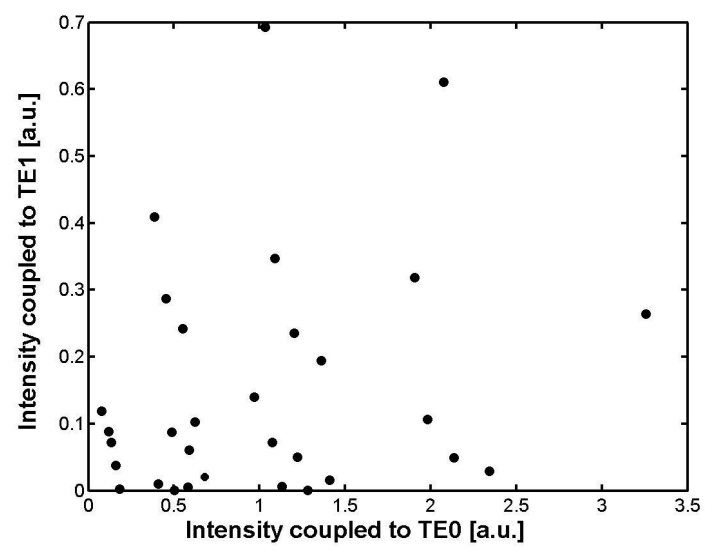

Fig. 5 A matrix of the power coupled to TE0- and TE1-modes from a set of asymmetric bit patterns, each of them having 5 pits. 


\section{PHASE DETECTION}

Phase of an optical signal can be measured using interferometric techniques. We have designed an integrated optical interferometer that can measure the phase difference between two points on the wavefront of an optical signal reflected from a disc. Fig. 6 shows a basic layout of a simple integrated optical phase detection system. It consists of two isolated single-mode waveguides connected to a multimode interference coupler with three output channels. Light from an illuminated bit pattern on a disc couples to the two channels and propagates to the coupler where the signals from the two waveguides interfere. By detecting and processing the signals from the three output channels, the phase difference between two parts of the field profile intercepted by two input channels can be obtained. The features of the different bit patterns can be distinguished by comparing the phase differences measured for each of them.

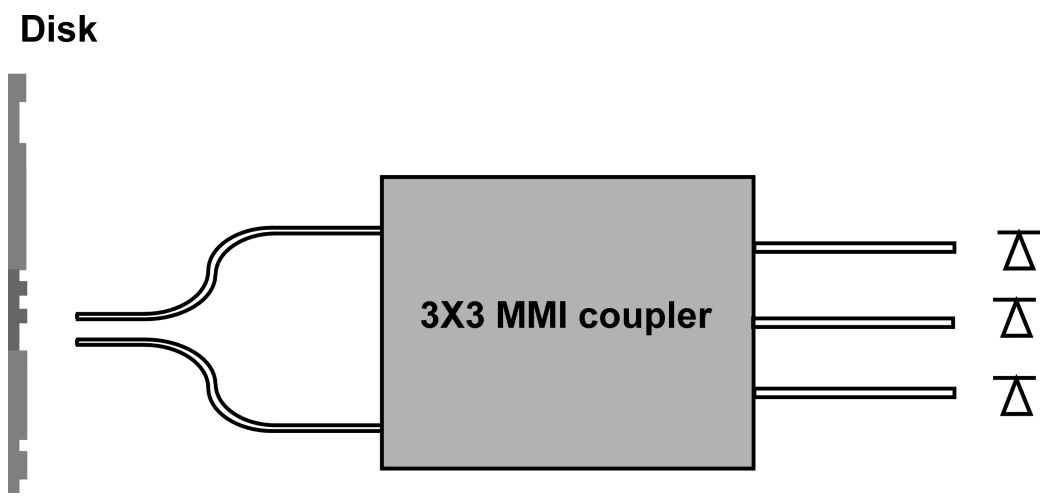

Fig. 6 Basic design of a simple interferometer comprising two input channels, a multimode interference (MMI) coupler and three output channels.

To get an impression of what can be achieved by this system we have calculated the phase difference between two parts of a wavefront originated from bit patterns on a disc intercepted by the two waveguides. In this calculation we have used the same asymmetric bit patterns of 5 pits as in subsection 5.1.2. Fig. 7 shows the phase difference calculated for the $2^{5}$ bit patterns. The results clearly indicate that for each bit pattern, a distinct phase difference is measured so that it can be used to encode the different bit patterns. It should be noted that theoretically there is no limit for the smallest phase difference that can be measured with the interferometer depicted in Fig. 6. The smallest phase difference that can be measured is determined be practical and experimental factors.

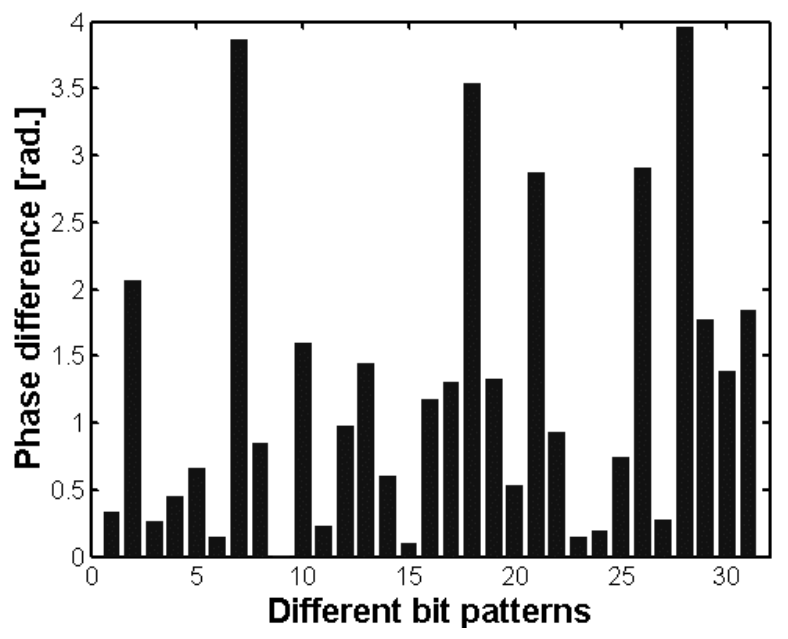

Fig. 7 A diagram comparing phase differences measured for 32 possible bit patterns from a 5-pit configuration. The pit lengths are 0.2 and $0.15 \lambda$ to the left and right of the center of the bit pattern, respectively. 


\section{CONCLUSIONS}

We have shown a read-out system incorporating optical waveguides that can be used to enhance the performance and resolution of optical disc. The far-field method exhibits performance better than that of the confocal microscope. Resolution beyond the diffraction limit can be achieved by intermediate-field detection. However, more rigorous calculations have to be done. Experiments to demonstrate these ideas are being performed.

\section{REFERENCES}

1. T.D. Milster, "Near-field optics: a new toll for data storage," Proceedings of IEEE, vol. 88, No. 9, pp. 1480-1490, September 2000.

2. F. Fransoo, D. Van Thourhout, L. Van Landschoot, S. Verstuyft, R. Baets, "A multimodal waveguide for enhanced performance in optical disc read-out", EOS Topical Meeting in Advanced Imaging Techniques 2003, pp. 115-117, Delft, The Netherlands, Oct. 2003.

3. S. F. Pereira, J.A. de Pooter, M. de Haan, and J.J.M. Braat, "Towards subwavelength optical detection using waveguides," Proceedings of the 7th LEOS Benelux symposium, pp. 28-31, Amsterdam, 2002.

4. W. Lukosz, J. Opt. Soc. Am., vol. 56, pp. 1463-1472, 1966.

5. M.I. Kolobov and C. Fabre, "Quantum limits on optical resolution," Phys. Rev. Lett., vol. 85, No. 18, pp. 37893792, 2000.

6. F. Fransoo, D. Van Thourhout, R. Baets, "Performance Analysis of a multi-mode waveguide based optical disc readout system," Applied Optics (to be published). 